\title{
Conceptual causal framework to assess the effect of SARS-CoV-2 variants on COVID-19 disease severity among hospitalized patients
}

Nina Van Goethem ${ }^{1,2^{*}+} \mathbb{D}$, Ben Serrien ${ }^{1 \dagger}$, Mathil Vandromme ${ }^{1}$, Chloé Wyndham-Thomas ${ }^{1}$, Lucy Catteau ${ }^{1}$, Ruben Brondeel ${ }^{1}$, Sofieke Klamer ${ }^{1}$, Marjan Meurisse ${ }^{1}$, Lize Cuypers ${ }^{3}$, Emmanuel André ${ }^{3,4}$, Koen Blot ${ }^{1}$ and Herman Van Oyen ${ }^{1}$

\begin{abstract}
Background: SARS-CoV-2 strains evolve continuously and accumulate mutations in their genomes over the course of the pandemic. The severity of a SARS-CoV-2 infection could partly depend on these viral genetic characteristics. Here, we present a general conceptual framework that allows to study the effect of SARS-CoV-2 variants on COVID19 disease severity among hospitalized patients.

Methods: A causal model is defined and visualized using a Directed Acyclic Graph (DAG), in which assumptions on the relationship between (confounding) variables are made explicit. Various DAGs are presented to explore specific study design options and the risk for selection bias. Next, the data infrastructure specific to the COVID-19 surveillance in Belgium is described, along with its strengths and weaknesses for the study of clinical impact of variants.

Discussion: A well-established framework that provides a complete view on COVID-19 disease severity among hospitalized patients by combining information from different sources on host factors, viral factors, and healthcarerelated factors, will enable to assess the clinical impact of emerging SARS-CoV-2 variants and answer questions that will be raised in the future. The framework shows the complexity related to causal research, the corresponding data requirements, and it underlines important limitations, such as unmeasured confounders or selection bias, inherent to repurposing existing routine COVID-19 data registries.
\end{abstract}

Trial registration: Each individual research project within the current conceptual framework will be prospectively registered in Open Science Framework (OSF identifier: https://doi.org/10.17605/OSF.IO/UEF29). OSF project created on 18 May 2021.

Keywords: COVID-19, SARS-CoV-2 variants, Hospitals, Causality

\footnotetext{
* Correspondence: Nina.VanGoethem@sciensano.be

${ }^{+}$Nina Van Goethem and Ben Serrien contributed equally to this work.

'Scientific Directorate of Epidemiology and public health, Sciensano, J.

Wytsmanstraat 14, 1050 Brussels, Belgium

${ }^{2}$ Department of Epidemiology and Biostatistics, Institut de recherche

expérimentale et clinique, Faculty of Public Health, Université catholique de Louvain, Clos Chapelle-aux-champs 30, 1200 Woluwe-Saint-Lambert, Belgium

Full list of author information is available at the end of the article
}

(c) The Author(s). 2021 Open Access This article is licensed under a Creative Commons Attribution 4.0 International License, which permits use, sharing, adaptation, distribution and reproduction in any medium or format, as long as you give appropriate credit to the original author(s) and the source, provide a link to the Creative Commons licence, and indicate if changes were made. The images or other third party material in this article are included in the article's Creative Commons licence, unless indicated otherwise in a credit line to the material. If material is not included in the article's Creative Commons licence and your intended use is not permitted by statutory regulation or exceeds the permitted use, you will need to obtain permission directly from the copyright holder. To view a copy of this licence, visit http://creativecommons.org/licenses/by/4.0/. The Creative Commons Public Domain Dedication waiver (http://creativecommons.org/publicdomain/zero/1.0/) applies to the data made available in this article, unless otherwise stated in a credit line to the data. 


\section{Background}

The pathogenesis of SARS-CoV-2 infection ranges from mild symptoms to severe respiratory failure [1]. Host factors play an important role in COVID-19 disease severity. Many studies have already identified older age and certain comorbidities, such as chronic immunocompromised conditions, chronic kidney disease, cardiovascular disease, diabetes mellitus, and obesity, as risk factors for hospitalization and mortality [2-5]. In addition, genetic association studies have identified several host genetic risk factors to become severely ill when infected by SARS-CoV-2 [6], including genetic variants in genes related to the immune system, such as the $\mathrm{Hu}$ man Leukocyte Antigens (HLA) gene complex [7] and cytokine genes, or in genes encoding human receptors of SARS-CoV-2 [8], such as ACE2 [9] and TMPRSS2 [10-12]. COVID-19 vaccines have proven to be highly effective against laboratory-confirmed SARS-CoV-2 infections and COVID-19 hospitalizations, severe disease, and deaths [13-19]. Whilst the vaccine effectiveness is shown at the population level, individual responses to vaccines will differ as a result of host factors and external factors $[20,21]$. Next to vaccination and other factors related to the host, severity of outcome can be influenced by aspects related to the healthcare organization and patient management $[5,22,23]$.

Finally, severity of a SARS-CoV-2 infection could depend on the viral genetic characteristics. For other viruses such as influenza, it is well documented that viral genetic variation plays an important role in pathogenicity [24-28]. SARS-CoV-2, as other RNA viruses, evolves continuously via point mutations, deletions, insertions and possibly re-assortments resulting in an expanding phylogenetic diversity. This genetic diversity can lead to the emergence of new variants with specific characteristics. Most emerging mutations will not provide a selective advantage to the virus, however some circulating variants may have increased viral fitness and are consequently labeled as 'Variant of Concern (VOC)' [29]. When the emerging variant possesses a selective advantage, it may dominate other circulating variants as time goes by [30]. Whole-genome sequencing (WGS) of the SARS-CoV-2 genome has been extensively applied during the COVID-19 pandemic [31, 32] and rapid public sharing of sequences allowed researchers to look at associations between SARS-CoV-2 genomic variants and disease severity [33-41]. However, these analyses have often remained inconclusive due to small sample sizes, sampling biases, limited availability of detailed patient data and the inability to appropriately adjust for potential confounding factors. Given these encountered limitations that are inherent to observational studies based on real-life data, making causal claims is challenging and requires transparency of assumptions and corrections through study design and statistical analyses. All factors listed above should be studied within one framework as they all influence disease outcomes among COVID-19 patients.

In this manuscript, we present a conceptual framework that allows to study the effect of SARS-CoV-2 variants on COVID-19 disease severity among hospitalized patients. First, we construct a causal model in a general hospital setting and identify important confounders, assess potential selection bias and state the underlying assumptions. Next, the causal model is translated into data needs and we describe the structure of the data collection that is specific to the surveillance of COVID-19 in Belgium, as well as the corresponding architecture linking the different data sources which allows to combine information on the viral genome, host characteristics, vaccination status, clinical outcomes, and other factors such as healthcare organization.

\section{Methods \\ Definition of exposure and outcome}

SARS-CoV-2 genetic variation can be defined on different levels, being SARS-CoV-2 lineages, clades, proteinlevel mutations, or single nucleotide polymorphisms (SNPs). WGS, or at least Sanger sequencing of selected parts of the viral genome, should be performed to confirm infection with a specific variant. Alternative methods, such as diagnostic PCR-based assays, can also be used as an indicator or screening method for particular VOCs. However, results from the latter should not be over-interpreted as they only identify specific mutations, and fail in definitive confirmation of a VOC or non-VOC variant [42]. The genetic variants of interest will change over the course of the epidemic; yet, the study protocol can and should stipulate $a$ priori hypotheses to avoid fishing expeditions. For simplicity, we will denote the exposure of interest as 'variant'. Similarly, the outcome 'severity' is a broad denominator that should be clearly defined before starting any analysis. Examples of severity indicators among hospitalized patients are admission to an intensive care unit, the use of invasive ventilation or extracorporeal life support (ECLS), acute respiratory distress syndrome (ARDS), length of stay in the hospital, or mortality.

\section{Description of the causal model}

We use a Directed Acyclic Graph (DAG) to explicitly state the underlying assumptions that are made to estimate the causal effect of infection with a SARS-CoV-2 variant on COVID-19 disease severity among hospitalized patients [43]. The DAG represents both observed and unobserved random variables. Arrows in the DAG denote direct causal effect, while the absence of an arrow between two variables represents the assumption of no 
causal effect. More details on causal inference and basic DAG concepts can be found elsewhere [43-45]. The DAG in Fig. 1 comprises our qualitative causal assumptions using background knowledge based on the literature and expert opinion.

The probability of infection with a SARS-CoV-2 variant depends among others on the age, gender, ethnicity, host genetics, immune status, comorbidities, and socioeconomic status (SES) of the patient [38, 46, 47]. These factors may also influence the severity of COVID-19 disease and can therefore be considered as common causes of the exposure-outcome relationship. Adjusting for these confounders is necessary in order to obtain an unbiased estimate of the effect of variant on severity. For example, if we assume that host genetics influence both the chance of being infected with a particular SARSCoV-2 variant and the chance to develop severe complications, omission of this variable could potentially invalidate the conclusions. In the reality of an observational design, we will be faced with unmeasured factors and we will not be able to adjust for all variables that we consider as potential confounders.

Further, we assume that the increased circulation of a SARS-CoV-2 variant within the general population might be associated with a peak in cases [47] and a subsequent surge in hospital admissions due to increased transmissibility and/or severity, or due to coincidence [40]. Consequently, the circulation of a SARS-CoV-2 variant and a high occupancy rate of hospital beds reserved for COVID-19 patients are possibly linked to the stage of the epidemic (time) within a given geographical region (place of residence) [48]. We learned from previous analyses that overflow of recognized intensive care unit (ICU) beds negatively impacts the outcome of the patient [5]. In order to estimate the causal effect of a SARS-CoV-2 variant on severity, we therefore need to

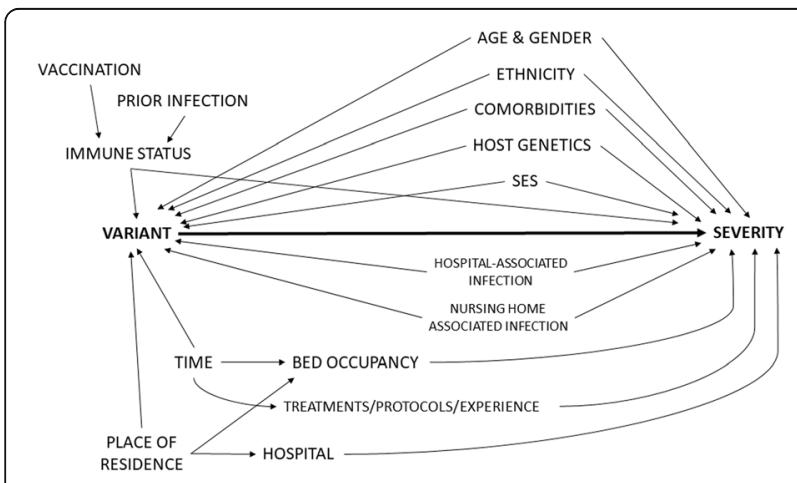

Fig. 1 Conceptual framework to assess the causal relationship between SARS-CoV-2 variants and COVID-19 disease severity among hospitalized patients presented in a Directed Acyclic Graph (DAG). VARIANT = infection with a particular SARS-CoV-2 variant. SEVERITY = developing severe complications following SARS-CoV-2 infection. $\mathrm{SES}=$ socio-economic status adjust the analysis for bed occupancy to avoid a spurious association between variant and severity that flows from variant via time or place of residence to bed occupancy and finally to severity.

In addition, the circulation of a variant can coincide in time with changes in treatment guidelines, protocols and experience. A spurious association between variant and severity can be prevented by adjusting for time, which could for example be defined by "wave". This will block the backdoor pathway from variant to treatments/protocols/experience through time to severity. Previous analyses also showed that patient outcomes significantly vary between hospitals $[5,49]$. Patients are more likely to be admitted to a hospital in close proximity to their place of residence. The increased circulation of a variant could coincide in place with the location of a hospital. Therefore, a spurious association between variant and severity should be prevented by adjusting for place or adjusting for the hospital where the patient is treated.

Similarly as for time and place of residence, the circulation of SARS-CoV-2 variants may differ between settings that are relatively disconnected from each other. The risk to be infected with a particular variant within a healthcare setting, such as a hospital or nursing home, is potentially different from the risk within the community. Healthcare-associated COVID-19 infections have been shown to be associated with higher risk of clinical deterioration (requirement of ventilatory support, critical care or death), likely because hospitalized patients represent a frailer population with decompensated comorbidities compared to the community [23]. Consequently, the analysis should be adjusted for infections contracted within the hospital or within a nursing home.

\section{Blocking non-causal associations}

After identifying the sufficient set of variables from the DAG, we can remove the bias resulting from nondirected open paths by covariate adjustment in a regression analysis and subsequently identify the targeted causal path between variant and severity. Alternatively, we can consider balanced matching to force the distribution of the matching factors to be identical across the exposed and unexposed groups. Figure 2 represents a DAG of a possible matched cohort design within the conceptual framework where the exposed and nonexposed are matched based on similar levels of care, i.e. hospital and bed occupancy. The variable $S$ indicates whether an individual in the source population is selected for the matched study. Most cohort matching starts with exposed individuals, i.e. those infected with an emerging SARS-CoV-2 variants, and subsequent selection of unexposed individuals with the same values for the matching values. As a results, the exposed are more likely to be selected into the matched sub-cohort 


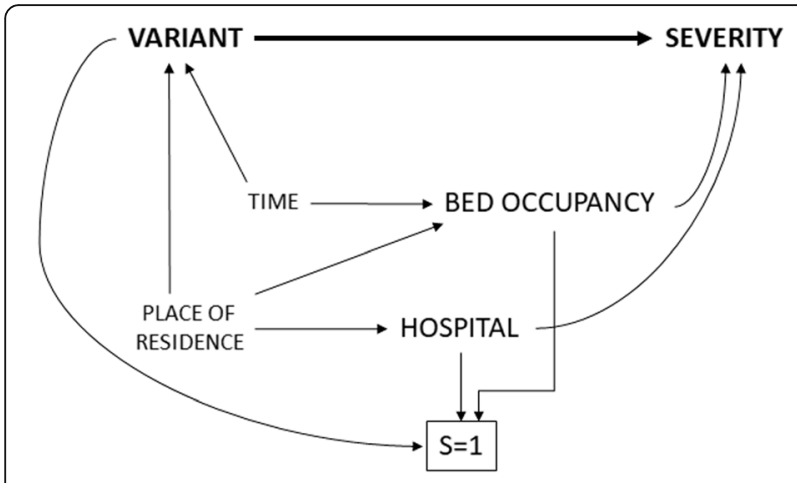

Fig. 2 Blocking non-causal associations between SARS-CoV-2 variants and COVID-19 disease severity among hospitalized patients by the use of a matched cohort design presented in a Directed Acyclic Graph (DAG). S = selection into the study

as represented by the arrow from variant to S. Unexposed individuals that are not matched to the exposed are usually discarded from the analysis (i.e. analysis is restricted to the matched subset) and hence the study sample has proportionally more patients exposed to the emerging variant than in the source population. The arrow from the bed occupancy and hospital to $S$ indicate that among the unexposed, individuals will be selected based on their values for bed occupancy and hospital. Matching (i.e. conditioning on $\mathrm{S}$ represented by a square box) induces an association via the path occupancy/hospital to $\mathrm{S}$ to variant that is of equal magnitude, but opposite direction, to the association via the path occupancy/hospital to variant (through time/place), ensuring that bed occupancy and hospital are both independent from variant in the matched sub-cohort. Because of the matching, the joint distribution of the matched data does not follow the causal structure for the source population as presented in the DAG, in the sense that the paths between variant and hospital and between variant and occupancy through time and place are no longer present in the matched sub-population (i.e. unfaithfulness [50]).

\section{Identification of selection bias}

Even in the scenario in which it would be possible to adjust the analysis for all important confounders as depicted in the DAG (Fig. 1), there can still arise a spurious association between variant and severity due to selection bias (Fig. 3). WGS is not performed on the samples of all COVID-19 patients, but on a selection of them. Subsequently, only patients with known exposure, i.e. information on the SARS-CoV-2 variant that they are infected with, will be included in the study. Figure 3A represents the situation in which the selection of samples for WGS depends on the severity of the patient, i.e. conditioning on the outcome, which is potentially problematic and is often denoted as sample truncation bias [51]. Severity is affected by the variant, as well as by other factors which are subsumed in an error term E. Indeed, severity is a collider variable (i.e. a common effect) on the path between variant and the error term $E$, and conditioning on severity induces a spurious association between variant and $E$ in the selected sample, even if they are independent in the general population. As such, restricting the sample to severe patients results in a newly induced non-causal path that flows from variant to E to severity. Figure 3B represents the situation when the total causal effect cannot be estimated in the case of conditioning (i.e. selection) on an intermediate variable. Due to analytic quality reasons it is recommended to

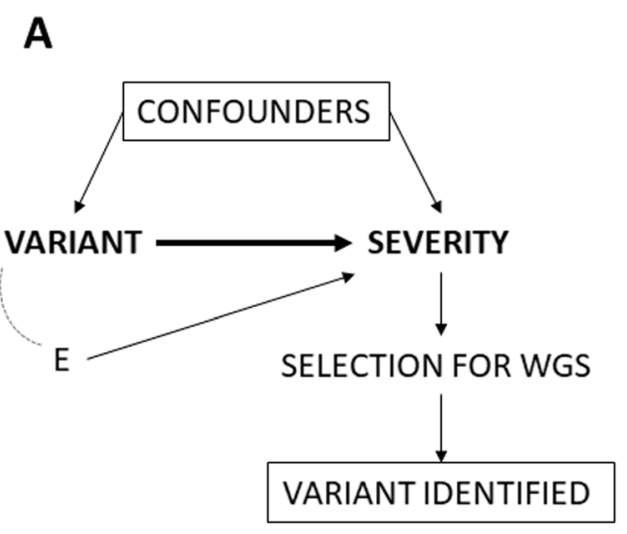

B

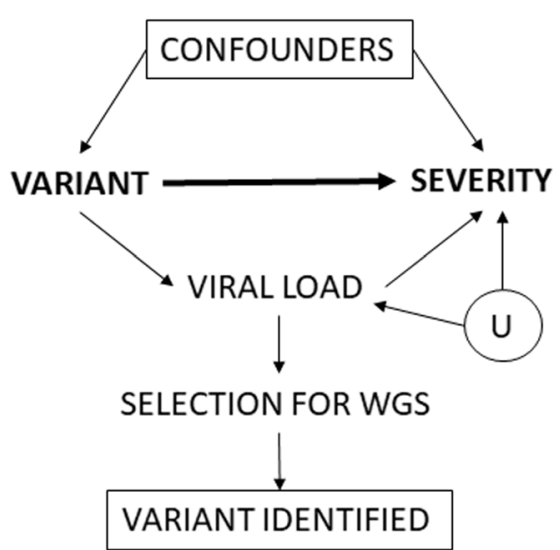

Fig. 3 Conceptual framework to assess the causal relationship between SARS-CoV-2 variants and COVID-19 disease severity among hospitalized patients presented in a Directed Acyclic Graph (DAG) in the scenario of selection bias. CONFOUNDERS = all other confounders as listed in the DAG in Fig. 1. $E=$ error term. $U=$ unmeasured confounders 
only select samples for sequencing with a sufficient high viral load $\left(\geq 10^{3}-10^{4}\right.$ RNA copies $\left./ \mathrm{mL}\right)$ [52]. Conditioning on samples with a high viral load blocks the causal path of variant on severity that is mediated by the viral load [53] leading to overcontrol bias [51]. In addition, conditioning on viral load may induce a spurious association between variant and severity when there exists an unmeasured confounder $(\mathrm{U})$ of the relationship between viral load (i.e. the mediator) and severity (i.e. the outcome).

\section{Translation into data requirements}

The causal model is translated into data requirements in order to meet the assumptions as depicted in the DAG (Fig. 1). In the context of the COVID-19 pandemic, Sciensano, the Belgian institute for health, has been mandated to describe the evolution of the epidemic and assess its consequences on the health of the Belgian population. Sciensano collects data on laboratoryconfirmed COVID-19 cases, testing, hospitalized COVID-19 patients, COVID-19 deaths and COVID-19 vaccinations. These surveillance systems have in common that they channel data flows from one or more sources that reach Sciensano either directly or via an intermediate step. Amongst others, Sciensano has launched the LINK-VACC project, which aims at linking selected variables from existing registries for COVID-19 vaccine surveillance. The IT architecture of the LINKVACC platform, hosted by the healthdata.be service of Sciensano, is used to organize data transfers to store and to link the different data sources based on the national registry number within a pseudonymized environment.
The present framework takes place within the architecture of the LINK-VACC project which allows to meet the assumptions of the causal model by combining selected variables from multiple data sources merged on the individual patient level (Fig. 4). The protocol of the LINK-VACC project was approved by the medical ethics committee University Hospital Brussels - Vrije Universiteit Brussel (VUB) on 03/02/2021 (reference number 2020/523) and obtained authorization from the Information Security Committee (ISC) Social Security and Health (reference number IVC/KSZG/21/034).

\section{Data on hospitalized COVID-19 patients}

Data on hospitalized COVID-19 patients is collected through two complementary surveillance systems [54]. The Surge Capacity Survey (SCS) exhaustively collects data on the number of COVID-19 patients at an aggregated level per hospital and enables calculation of the daily occupancy rate of beds reserved for COVID-19 patients per hospital accreditation number. The Clinical Hospital Survey (CHS) collects individual data of patients hospitalized with confirmed COVID-19 through an admission, discharge and detailed ICU form. The $\mathrm{CHS}$ is not exhaustive as participation is voluntary and covers approximately $65 \%$ of all hospitalized COVID-19 patients in Belgium. However, the CHS is considered to be representative as it covers all provinces in Belgium and includes public and private, academic and nonacademic hospitals.

II. Data on COVID-19 test results

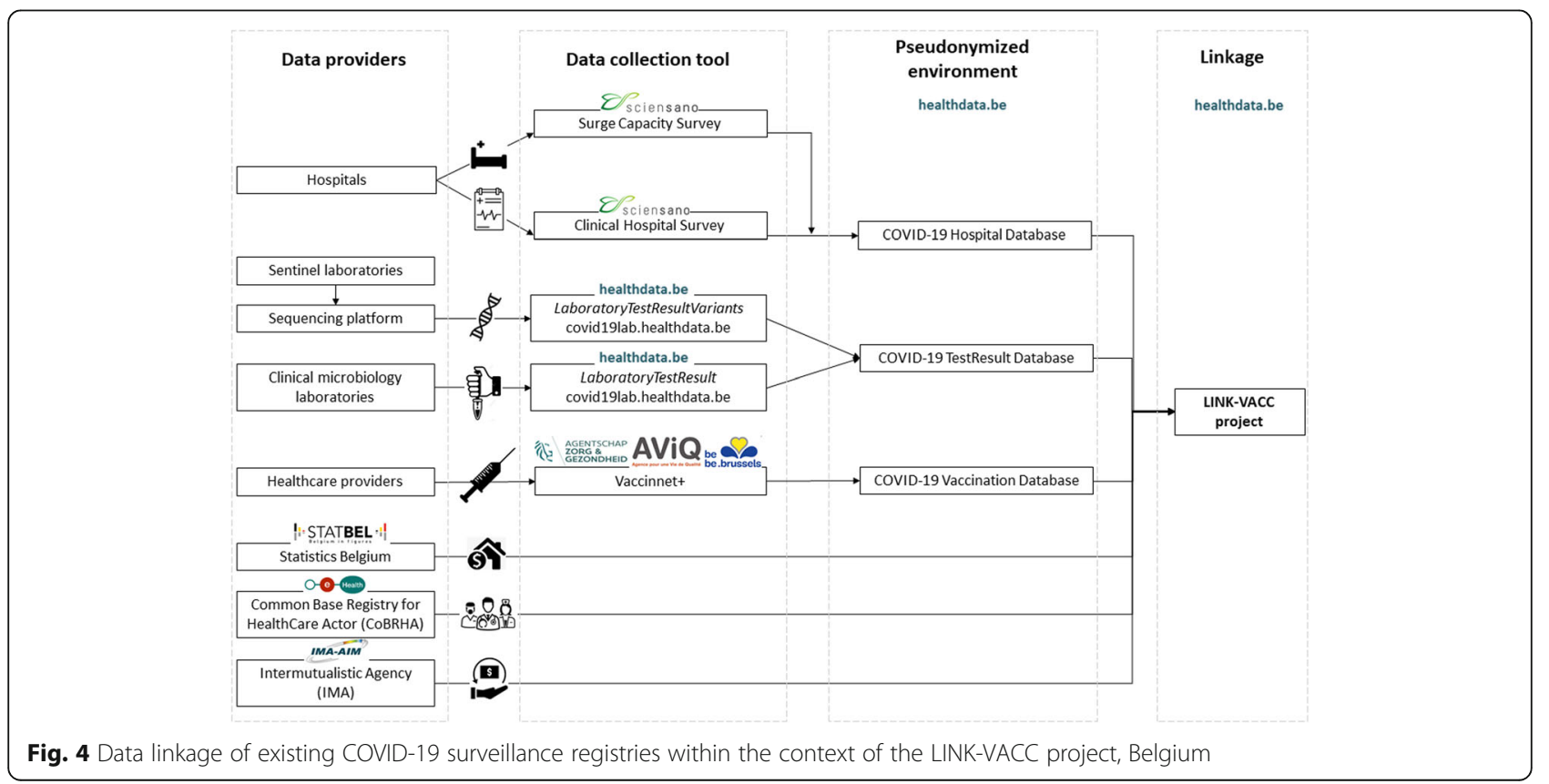


The COVID-19 TestResult Database, hosted by the Sciensano service Healthdata.be [55], collects the RTPCR and antigen test results from clinical microbiology laboratories (CML) and physicians since the 5th of May 2020, including retrospective data collection since February 2020 [56]. The test results are accompanied by the date of sampling, the date of test result, the test result, the type of diagnostic test, a sample identification number, the laboratory identification number and patient demographic variables. Daily reporting of all test results of RT-PCR and antigenic diagnostic tests is mandatory for reimbursement.

\section{Data on COVID-19 sequencing results}

The National Reference Center (NRC) of respiratory diseases has put in place genomic surveillance at the national level since the first introduction of the virus, together with other university centers. The genomic surveillance of SARS-CoV-2 has scaled-up from December 2020 onwards resulting in a federal sequencing consortium currently including a total of 17 laboratories. Given the emergence of VOCs in December 2020, the samples selected for sequencing at that time resulted mainly from active surveillance focusing on returning travelers, atypical PCR results and large outbreaks. Subsequently, baseline surveillance was set up in January 2021 to obtain a representative sample of the positive cases. This is obtained through collaboration with a sentinel network of laboratories that send a proportion of their positive samples to one of the laboratories that are part of the sequencing consortium to ensure an optimal geographical coverage and a diversity of clinical severity patterns. The aim is to cover approximately $10 \%$ of all positive cases in Belgium. The baseline surveillance is complemented by targeted active surveillance that focuses on the systematic screening of patients experiencing re-infection, vaccine breakthrough cases, immunocompromised patients, and a selection of samples linked to outbreaks, returning travelers from red zones and atypical PCR results [52]. In addition, hospitals and laboratories are allowed to perform additional sequencing outside reimbursement indications on own initiative: some hospitals took the initiative to systematically sequence all available positive samples from patients admitted to ICU, while other hospitals aim to sequence exhaustively all available SARS-CoV-2 positive samples from their hospitalized patients.

The reporting of variant information from sequencing laboratories to Sciensano through h-Healthdata.be has been put in place since March 2021. For this purpose, an additional message ("LaboratoryTestResultVariants") is to be sent to the Central COVID-19 Database [57]. After sequencing, the SARS-CoV-2 variants are assigned a Pangolin lineage, the nomenclature system for SARS$\mathrm{CoV}-2$ that has been put in place by Rambaut et al [58], and are subsequently designated as confirmed SARS$\mathrm{CoV}-2$ variants. Besides information on confirmed variants obtained through sequencing, the laboratories also report test results from diagnostic SNP assays to detect samples compatible with known VOCs. The collected information consists of the indication of the reason for which the sample was selected for sequencing (baseline or active surveillance), the mutations tested in case of an RT-PCR SNP assay, the mutations and deletions detected in the $\mathrm{S}$ gene, and in case of sequencing: the Pangolin lineage and the GISAID accession number.

\section{Data on COVID-19 vaccines}

As defined by the Belgian law, all COVID-19 vaccines administered in Belgium are recorded in Vaccinnet+, the national vaccine registry. Vaccination data are subsequently sent to Healthdata.be at Sciensano. Researchers at Sciensano have access to a pseudonymized version of these data including demographic data of the vaccinated person (age, gender and place of residence) and information on the administered vaccine (brand, lot number, administration date and registration date).

\section{Other data sources}

Within the LINK-VACC environment, data from existing registries outside Sciensano will also be linked on the individual patient level using the national registry number. Statbel, the Belgian statistical office, collects and shares data on the Belgian economy, society and territory. Individually-linked data will allow to retrieve socio-economic information (e.g. civil status, employment status and income decile), identify residents of collectivities, and obtain information on long-term survival and cause of death. The Intermutualistic Agency (IMA) collects data on reimbursed care and medications of citizens insured in Belgium and will enable to provide information on comorbidities and pregnancy status, but also give insights on long-term healthcare and medication consumption (e.g. after COVID-19 hospitalization). The Common Base Registry for HealthCare Actor (CoBRHA) enables to identify Belgian healthcare workers by profession type based on their license to practice.

\section{Study population}

The study population consists of hospitalized COVID-19 patients with an available admission form registered in the CHS and with information available on the SARSCoV-2 variant (exposure) of their infection as obtained through the linkage of the CHS database with the COVID-19 TestResult Database (Fig. 5). The choice to 


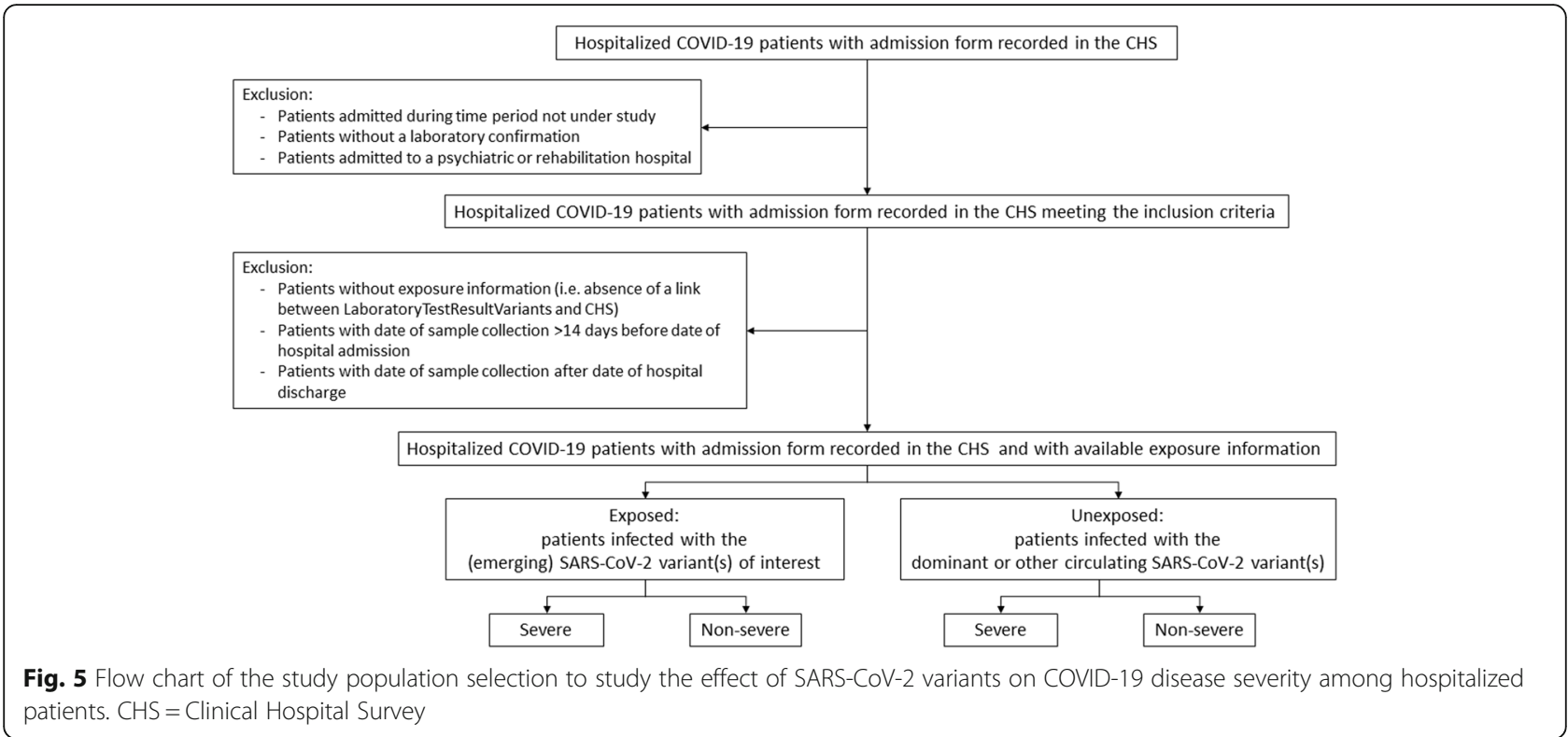

study severity among hospitalized patients is supported by the detailed information on patient characteristics and the well-defined severity indicators available in the CHS data collection. Patient information related to positive cases is limited to demographics (age, gender, and residence) and is not suitable to study severity of disease. In addition, defining severity based on hospitalization itself is hampered by the fact that information on hospital admission is derived from the non-exhaustive registration of hospitalized COVID-19 patients in the CHS. As a consequence, absence of registration of a patient in the $\mathrm{CHS}$ does not imply that this patient was not admitted to the hospital.

\section{Study design}

The study is an observational multi-center cohort study where COVID-19 hospitalized patients are followed-up from hospital admission until death or hospital discharge and for whom information is obtained by merging different national surveillance systems based on the national registry number. Within the described framework, several research questions, study designs and analyses are possible and should be defined on a case-by-case basis depending on the requirements at a specific time of the epidemic.

\section{Data analysis plan}

The definition of the outcome will define the statistical model being used. A logistic regression or log-binomial model may be used for binary outcomes and a survival analysis for time-to-event data. Selection of main effects in the model will be based on the minimal sufficient adjustment set of covariates as identified from the DAG. The model with all main effects will be compared to models including interaction terms between the exposure and covariates or including non-linear terms for continuous covariates. Regression standardization may be used to estimate the causal effects of interest. Alternative to standardization, we may use inverse-probability weighting and doubly-robust methods [43]. The modeling approach should account for the clustering effects within hospitals, for example by matching on hospital or by using generalized estimating equation (GEE) models. Sensitivity analyses can be used to assess robustness to unmeasured confounding, selection bias or measurement error [59]. If a matching procedure is used, the matching factors do not require adjustment in the model as they are already accounted for by the design. Multiple imputation will be used for missing data on the outcome or potential confounders. As the emergence of VOCs in Belgium occurred from December 2020 onwards, patients admitted before 1/12/ 2020 are classified as being exposed to "previously circulating SARS-CoV-2 variants". Vaccination rollout began in January 2021 and initially focused on nursing home residents and healthcare workers. From March 2021 onwards, people were vaccinated according to their age group. Therefore, patients admitted in the year 2020 are classified as non-vaccinated. A detailed data analysis plan will be anticipated for each specific research question addressing a particular exposure-outcome relationship. Each individual research project will be registered in Open Science Framework (https://osf.io/zg3dj)

\section{Discussion}

This manuscript presents a conceptual framework to study the effect of SARS-CoV-2 variants on COVID-19 
disease severity among hospitalized patients. A DAG was used to explicitly state the underlying assumptions of the causal model. Next, the DAG was translated into the data requirements, i.e. the necessary information on important confounders, that would allow to obtain an unbiased estimate of the causal effect of SARS-CoV-2 variants on severity of COVID-19 disease. The current framework takes advantage of the LINK-VACC architecture to combine information from existing surveillance registries on the viral genome, host characteristics, vaccination status, clinical outcomes, and other factors such as healthcare organization. As such, it allows to perform a cohort study among hospitalized COVID-19 patients, for whom information on the exposure, outcome and confounders is obtained by merging existing national surveillance systems based on the national registry number, to study the clinical impact of SARS-CoV-2 variants. Within the described framework, several research questions, study designs and analyses are possible and should be defined on a case-by-case basis depending on the requirements at a specific time of the epidemic.

The conceptual framework underlines important limitations of the current data architecture. First, not all confounders are correctly measured or even measured at all and the resulting bias should be quantified to assess robustness of the conclusions [59]. Host genetic variants may partially account for the residual variability in disease severity between patients after adjusting for other risk factors such as age and comorbidities $[12,60]$. Further, it is not unlikely that host genetic variants influence the probability to be infected with a particular SARS-CoV-2 variant given the importance of hostpathogen interactions [61]. Collecting information on the ethnicity of the patient may partially account for this bias [62-64], but residual confounding is likely to exist. Genetic profiling to discover patients' increased susceptibility to life-threatening conditions caused by an infectious disease such as COVID-19 has been put forward as one of the priorities in the 1+ Million Genomes Roadmap. However, integration of omics data in public health remains limited given the challenges related to the generation, analysis and interpretation of high-dimensional data [65] and potential privacy and security concerns. Next to the absence of information on confounders that should be adjusted for, the analysis can also suffer from inaccurate measurements or partial information on certain confounders. For example, the occupancy rate of ICU beds is currently calculated based on the number of COVID-19 patients in the hospital and the number of recognized ICU beds available in the hospital reserved for COVID-19 patients. However, the latter also depends on the load of non-COVID-19 patients which may vary between hospitals and the different waves according to the directives in force. Information on the number of
non-COVID-19 patients and their impact on total hospital or ICU capacity is less well documented. In addition, quality of care not only depends on occupancy rate, but also on the staff-to-patient ratio and the professional experience of healthcare workers.

Second, a potential threat for the current conceptual framework is the selection bias that arises when there is an increased focus on selecting samples for WGS from patients that have developed severe complications. As a consequence, the selection bias will have a strong effect on the representativeness as the study population will not represent the full spectrum of hospitalized COVID19 patients. In addition, selection bias can induce collider bias (which occurs after conditioning on a common effect) which can lead to substantially biased estimates of associations [66]. This underlines the importance of obtaining a representative and unbiased SARS-CoV-2 sequence collection. Both the European Centre for Disease Prevention and Control (ECDC) [67] and the World Health Organization (WHO) [68] have provided guidance on representative sampling and sequencing of SARS-CoV-2 cases from routine surveillance. In Belgium, genomic surveillance consists of a passive component that aims to obtain a representative set of sequences based on a network of sentinel laboratories, and an active component, including targeting sequencing of vaccine breakthrough cases and a selection of samples from outbreaks and returning travelers. In addition, there has been an increased focus on sequencing samples from COVID-19 patients admitted to an intensive care unit. The data collection tool contains a variable "indication for WGS" which allows to differentiate between samples obtained from the active and passive surveillance arm and can hence be used to eliminate the selection bias. Even if there is no selection bias among the hospitalized patients, the current framework only allows to estimate the risk associated with a particular SARS-CoV-2 variant on severe clinical evolution once hospitalized. Estimating the effect of SARS-CoV-2 variants on disease severity among the general population of COVID-19 patients requires linkage with an exhaustive data source of hospital admission data based on the national registry number.

Here, we have established a framework to study the exposure-outcome relationship by merging data from routine national surveillance systems. Observational studies that aim to estimate a causal effect are often faced with an imbalance of baseline characteristics of patients between groups. Adjustment for confounding variables can be accomplished by including them in a regression model or by conducting a matched cohort study [69]. Matching aims to balance the groups with respect to factors which may influence the outcome. However, if the matching ratio is low, the matching design 
may suffer from loss of efficiency as the analysis is restricted to a subset of patients. Also, if interaction effects between the exposure and covariates are of interest and the objective of the study, these covariates cannot be used in the matching criterion.

The study population consists of a cohort of hospitalized COVID-19 patients that are registered in the CHS and with available SARS-CoV-2 variant information. The sample size is therefore limited by the presence of the patient in the two independent data sources. The advantage of this strategy is that re-using existing data sources avoids investing resources in setting up additional data collection systems. However, the ability to provide an answer to our research questions heavily depends on the features of the existing data sources. Given that the data collection system was not specifically designed for the requirements of the current analysis, it may result in potential threats to the causal model of interest, such as unmeasured confounding and selection bias. However, the anticipation of future research questions is a challenging task as research interests typically change over the course of a pandemic. This underlines the importance of the establishment of a versatile framework that allows researchers to efficiently combine data from different sources and which is flexible to be adjusted for different purposes. An alternative approach for the selection of samples for sequencing that would circumvent the selection bias and the relatively small sample size related to linkage of existing data sources is to conduct a nested case-control study within the cohort of hospitalized patients registered in the CHS. Several methods to sample controls can be applied. For example, one can randomly select a control each time a case is diagnosed. Another possibility is to sample the control group at the beginning of the follow-up period resulting in a sample that is representative of the full cohort from which all future cases will develop. This type of nested case-control study is usually referred to as a case-cohort study. If the controls are indeed a representative sample of the study base, the exposure odds ratio is a valid estimate of the incidence rate ratio one would obtain from a cohort study. In addition, a case-cohort design has the advantage that a single control group can be applied for multiple outcomes. A careful selection of cases and controls and the subsequent sequencing of the samples of these patients allows an efficient use of resources. Indeed, a nested case-control study design is particularly appealing when the assessment of the exposure is expensive. However, a retrospective selection for WGS requires the preservation of samples, i.e. by bio-banking or mid-term storage of the samples of all hospitalized patients.

As a final remark, statistical analyses under the proposed causal model require considerable sample sizes which can take a while to accumulate while a new variant emerges. After the arrival of a new variant, it takes several weeks before it becomes dominant and/or before it reaches the more vulnerable population that has a high risk of hospitalization. In addition, we should take into account the length of stay and reporting delay before the hospital discharge data is recorded in the surveillance system. As a consequence, rapid assessments of the clinical impact of new emerging SARS-CoV-2 variants among hospitalized patients is challenging. The uncertainty related to small sample sizes in the early phases of a new emerging variant should be acknowledged by researchers and policy makers in their communication to the public.

\section{Conclusion}

A well-established framework that brings together information from different domains and thereby provides a complete view on the factors that influence COVID-19 disease severity will enable to assess the impact of emerging SARS-CoV-2 variants and answer questions that will be raised in the future. This conceptual framework is important as a theoretical foundation for the development of routine clinical epidemiological research and may serve as a basis for future pandemics. An evaluation and update of the framework should be conducted regularly in terms of emerging new viral, social or clinical trends or when a new data architecture allows for improved analyses.

\section{Abbreviations}

ACE2: Angiotensin converting enzyme 2; ARDS: Acute respiratory distress syndrome; CHS: Clinical hospital survey; CML: clinical microbiology laboratories; CoBRHA: Common Base Registry for HealthCare Actor; COVID19: Coronavirus disease 2019; DAG: Directed acyclic graph; ECDC: European Centre for Disease Prevention and Control; ECLS: Extracorporeal life support; GDPR: General Data Protection Regulation; GEE: Generalized estimating equation; GISAID: Global initiative on sharing all influenza data; HLA: Human Leukocyte Antigens; ICU: Intensive Care Unit; IMA-AIM: Intermutualistic Agency; ISC: Information Security Committee; IT: Information Technology; LINK-VACC: Linking of registries for COVID-19 vaccine surveillance; NRC: National Reference Center; RNA: Ribonucleic Acid; RT-PCR: Reverse Transcriptase-Polymerase Chain Reaction; SARS-CoV-2: severe acute respiratory syndrome coronavirus 2; SCS: Surge Capacity Survey; SNP: Single Nucleotide Polymorphism; TMPRSS2: Transmembrane Serine Protease 2; VOC: Variant of Concern; WGS: Whole-Genome Sequencing; WHO: World Health Organization

\section{Acknowledgements}

We would like to sincerely thank all hospitals taking part in the surveillance and providing valuable information about hospitalized COVID-19 patients, greatly contributing to the management of COVID-19 in Belgium. We would like to thank all people in charge of the clinical microbiology laboratories for their collaboration and transfer of data. We would like to thank Johan Van Bussel, Kurt Vanbrabant, Andreas Gryncewicz, and other colleagues of Healthdata.be to set up the data infrastructure. Finally, we would like to thank Dieter Van Cauteren, Freek Haarhuis, and other colleagues at Sciensano for their valuable insights and feedback.

Authors' contributions

Conceptualization: N.V.G., B.S., M.V., K.B.; Project Administration: L.C., C.W.T.; Writing - Original Draft Preparation: N.V.G., B.S.; Writing - Review \& Editing: 
all authors; Supervision: H.V.O. The author(s) read and approved the final manuscript.

\section{Funding}

No specific funding for this study.

\section{Availability of data and materials}

The data obtained through the surveillance systems are available from the corresponding author on reasonable request according to Sciensano scientific policy and after approval by the Belgian data protection authority.

\section{Declarations}

\section{Ethics approval and consent to participate}

The protocol of the LINK-VACC project obtained authorization from the Information Security Committee (ISC) Social Security and Health (reference number IVC/KSZG/21/034) and was approved by the medical ethics committee UZ Brussel - VUB on 03/02/2021 (reference number 2020/523). The protocol of the COVID-19 Clinical Hospital Survey project was approved by the medical ethics committee University Hospital Ghent on 24/03/2020 and obtained authorization from the Information Security Committee (ISC) Social Security and Health (reference number IVC/KSZG/20/038).

\section{Consent for publication}

Not applicable.

\section{Competing interests}

Herman Van Oyen is editor of Archives of Public Health. The other authors declare that they have no competing interests.

\section{Author details}

${ }^{1}$ Scientific Directorate of Epidemiology and public health, Sciensano, J. Wytsmanstraat 14, 1050 Brussels, Belgium. ${ }^{2}$ Department of Epidemiology and Biostatistics, Institut de recherche expérimentale et clinique, Faculty of Public Health, Université catholique de Louvain, Clos Chapelle-aux-champs 30, 1200 Woluwe-Saint-Lambert, Belgium. ${ }^{3}$ Department of Laboratory Medicine, National Reference Centre for Respiratory Pathogens, University Hospitals Leuven, Herestraat 49, BE-3000 Leuven, Belgium. ${ }^{4} \mathrm{KU}$ Leuven, Department of Microbiology, Immunology and Transplantation, Rega Institute for Medical Research, Laboratory Clinical Bacteriology and Mycology, Herestraat 49, box 1040, BE-3000 Leuven, Belgium.

\section{Received: 1 August 2021 Accepted: 2 October 2021}

Published online: 25 October 2021

\section{References}

1. Hu B, Guo H, Zhou P, Shi Z-L. Characteristics of SARS-CoV-2 and COVID-19. Nat Rev Microbiol. 2021;19(3):141-54. https://doi.org/10.1038/s41579-02000459-7.

2. Killerby ME, Link-Gelles R, Haight SC, Schrodt CA, England L, Gomes DJ, et al. Characteristics Associated with hospitalization among patients with COVID-19 - metropolitan Atlanta, Georgia, march-April 2020. MMWR Morb Mortal Wkly Rep. 2020;69(25):790-4. https://doi.org/10.15585/mmwr.mm692 5e1.

3. Kaeuffer C, Hyaric CL, Fabacher T, Mootien J, Dervieux B, Ruch Y, et al. Clinical characteristics and risk factors associated with severe COVID-19: prospective analysis of 1,045 hospitalised cases in north-eastern France, march 2020. Eurosurveillance. 2020;25(48):2000895.

4. Sim BLH, Chidambaram SK, Wong XC, Pathmanathan MD, Peariasamy KM, Hor CP, et al. Clinical characteristics and risk factors for severe COVID-19 infections in Malaysia: A nationwide observational study. Lancet Reg Health West Pac. 2020;4 [cited 2021 Mar 1]. Available from: https:/www.thelancet. com/journals/lanwpc/article/PIIS2666-6065(20)30055-9/abstract.

5. Taccone FS, Van Goethem N, Depauw R, Wittebole X, Blot K, Vanoyen H, et al. The role of organizational characteristics on the outcome of COVID-19 patients admitted to the ICU in Belgium. Lancet Reg Health Eur. 2020;23: 100019. https://doi.org/10.1016/j.lanepe.2020.100019.

6. Callaway E. The quest to find genes that drive severe COVID. Nature. 2021 595(7867):346-8. https://doi.org/10.1038/d41586-021-01827-w.

7. Shkurnikov M, Nersisyan S, Jankevic T, Galatenko A, Gordeev I, Vechorko V, et al. Association of HLA Class I Genotypes With Severity of Coronavirus
Disease-19. Front Immunol. 2021;12 [cited 2021 Jun 11]Available from: https:/www.frontiersin.org/articles/10.3389/fimmu.2021.641900/full.

8. Hou Y, Zhao J, Martin W, Kallianpur A, Chung MK, Jehi L, et al. New insights into genetic susceptibility of COVID-19: an ACE2 and TMPRSS2 polymorphism analysis. BMC Med. 2020;18(1):216. https://doi.org/10.1186/ s12916-020-01673-z

9. Benetti E, Tita R, Spiga O, Ciolfi A, Birolo G, Bruselles A, et al. ACE2 gene variants may underlie interindividual variability and susceptibility to COVID19 in the Italian population. Eur J Hum Genet. 2020;28(11):1602-14. https:// doi.org/10.1038/s41431-020-0691-z.

10. Elhabyan A, Elyaacoub S, Sanad E, Abukhadra A, Elhabyan A, Dinu V. The role of host genetics in susceptibility to severe viral infections in humans and insights into host genetics of severe COVID-19: a systematic review. Virus Res. 2020;289:198163. https://doi.org/10.1016/j.virusres.2020.198163.

11. Zeberg H, Pääbo S. A genomic region associated with protection against severe COVID-19 is inherited from Neandertals. PNAS. 2021;118(9) [cited 2021 Jun 11]. Available from: https://www.pnas.org/content/118/9/e20263 09118.

12. Fricke-Galindo I, Falfán-Valencia R. Genetics Insight for COVID-19 Susceptibility and Severity: A Review. Front Immunol. 2021;12 [cited 2021 May 21]. Available from: https://www.frontiersin.org/articles/10.3389/fimmu.2021.622176/full.

13. Haas EJ, Angulo FJ, McLaughlin JM, Anis E, Singer SR, Khan F, et al. Impact and effectiveness of mRNA BNT162b2 vaccine against SARS-CoV-2 infections and COVID-19 cases, hospitalisations, and deaths following a nationwide vaccination campaign in Israel: an observational study using national surveillance data. Lancet. 2021;397(10287):1819-29. https://doi. org/10.1016/50140-6736(21)00947-8.

14. Levine-Tiefenbrun M, Yelin I, Katz R, Herzel E, Golan Z, Schreiber L, et al. Initial report of decreased SARS-CoV-2 viral load after inoculation with the BNT162b2 vaccine. Nat Med. 2021;27(5):790-2. https://doi.org/10.1038/s41 591-021-01316-7.

15. Voysey M, Clemens SAC, Madhi SA, Weckx LY, Folegatti PM, Aley PK, et al. Safety and efficacy of the ChAdOx1 nCoV-19 vaccine (AZD1222) against SARS-CoV-2: an interim analysis of four randomised controlled trials in Brazil, South Africa, and the UK. Lancet. 2021 Jan 9;397(10269):99-111. https://doi. org/10.1016/S0140-6736(20)32661-1.

16. Baden LR, El Sahly HM, Essink B, Kotloff $K$, Frey $S$, Novak R, et al. Efficacy and safety of the mRNA-1273 SARS-CoV-2 vaccine. N Engl J Med. 2021;384(5): 403-16. https://doi.org/10.1056/NEJMoa2035389.

17. Vasileiou E, Simpson CR, Shi T, Kerr S, Agrawal U, Akbari A, et al. Interim findings from first-dose mass COVID-19 vaccination roll-out and COVID-19 hospital admissions in Scotland: a national prospective cohort study. Lancet. 2021;397(10285):1646-57. https://doi.org/10.1016/S0140-6736(21)00677-2.

18. Bernal JL, Andrews N, Gower C, Robertson C, Stowe J, Tessier E, et al. Effectiveness of the Pfizer-BioNTech and Oxford-AstraZeneca vaccines on covid-19 related symptoms, hospital admissions, and mortality in older adults in England: test negative case-control study. BMJ. 2021;373. https:// doi.org/10.1136/bmj.n1088

19. Nunes B, Rodrigues AP, Kislaya I, Cruz C, Peralta-Santos A, Lima J, et al. mRNA vaccine effectiveness against COVID-19-related hospitalisations and deaths in older adults: a cohort study based on data linkage of national health registries in Portugal, February to August 2021. Eurosurveillance. 2021;26: 2100833. https://doi.org/10.2807/1560-7917.ES.2021.26.38.2100833.

20. Zimmermann $\mathrm{P}$, Curtis $\mathrm{N}$. Factors That Influence the Immune Response to Vaccination. Clin Microbiol Rev. 2019;32(2) [cited 2021 Jun 12]. Available from: https://www.ncbi.nlm.nih.gov/pmc/articles/PMC6431125/.

21. Yelin I, Katz R, Herzel E, Berman-Zilberstein T, Ben-Tov A, Kuint J, et al. Associations of the BNT162b2 COVID-19 vaccine effectiveness with patient age and comorbidities. medRxiv. 2021;10.1101/2021.03.16.21253686v2.

22. Bravata DM, Perkins AJ, Myers LJ, Arling G, Zhang Y, Zillich AJ, et al. Association of intensive care unit patient load and demand with mortality rates in US Department of veterans affairs hospitals during the COVID-19 pandemic. JAMA Netw Open. 2021;4(1):e2034266. https://doi.org/10.1001/ja manetworkopen.2020.34266.

23. Gupta S, Hayek SS, Wang W, Chan L, Mathews KS, Melamed ML, et al. Factors Associated with death in critically ill patients with coronavirus disease 2019 in the US. JAMA Intern Med. 2020;180(11):1436-47. https://doi. org/10.1001/jamainternmed.2020.3596.

24. Freitas ARR, Donalisio MR. Excess of mortality in adults and elderly and circulation of subtypes of influenza virus in southern Brazil. Front Immunol. 2017;8:1903. 
25. Martínez A, Soldevila N, Romero-Tamarit A, Torner N, Godoy P, Rius C, et al. Risk factors associated with severe outcomes in adult hospitalized patients according to influenza type and subtype. PLoS One. 2019;14(1):e0210353. https://doi.org/10.1371/journal.pone.0210353.

26. Su S, Chaves SS, Perez A, D'Mello T, Kirley PD, Yousey-Hindes K, et al. Comparing clinical characteristics between hospitalized adults with laboratory-confirmed influenza a and B virus infection. Clin Infect Dis. 2014; 59(2):252-5. https://doi.org/10.1093/cid/ciu269.

27. Wedde M, Wählisch S, Wolff T, Schweiger B. Predominance of HA-222D/G polymorphism in influenza a(H1N1)pdm09 viruses associated with fatal and severe outcomes recently circulating in Germany. PLoS One. 2013;8(2): e57059. https://doi.org/10.1371/journal.pone.0057059.

28. Simon B, Pichon M, Valette M, Burfin G, Richard M, Lina B, et al. Whole genome sequencing of a(H3N2) influenza viruses reveals Variants Associated with severity during the $2016^{-} 2017$ season. Viruses. 2019;28:11(2).

29. European Centre for Disease Prevention and Control. Risk related to the spread of new SARS-CoV-2 variants of concern in the EU/EEA. 2020.

30. Torjesen I. Covid-19: Delta variant is now UK's most dominant strain and spreading through schools. BMJ. 2021;373:n1445. https://doi.org/10.1136/ bmj.n1445.

31. Alm E, Broberg EK, Connor T, Hodcroft EB, Komissarov AB, Maurer-Stroh S, et al. Geographical and temporal distribution of SARS-CoV-2 clades in the WHO European region, January to June 2020. Eurosurveillance. 2020;25(32): 2001410.

32. Wawina-Bokalanga T, Martí-Carreras J, Vanmechelen B, Bloemen M, Wollants E, Laenen L, et al. Genetic diversity and evolution of SARS-CoV-2 in Belgium during the first wave outbreak. bioRxiv. 2021;10.1101/2021.06.29.450330.

33. Voss JD, Skarzynski M, McAuley EM, Maier EJ, Gibbons T, Fries AC, et al. Variants in SARS-CoV-2 Associated with Mild or Severe Outcome. Evol Med Public Health. 2021;9(eoab019) [cited 2021 Jul 9]. Available from:267-75. https://doi.org/10.1093/emph/eoab019.

34. Nagy Á, Pongor S, Győrffy B. Different mutations in SARS-CoV-2 associate with severe and mild outcome. Int J Antimicrob Agents. 2021;57(2):106272. https://doi.org/10.1016/j.ijantimicag.2020.106272.

35. Nakamichi K, Shen JZ, Lee CS, Lee A, Roberts EA, Simonson PD, et al. Hospitalization and mortality associated with SARS-CoV-2 viral clades in COVID-19. Sci Rep. 2021;11(1):4802. https://doi.org/10.1038/s41598-021-82 850-9.

36. Toyoshima Y, Nemoto K, Matsumoto S, Nakamura Y, Kiyotani K. SARS-CoV-2 genomic variations associated with mortality rate of COVID-19. J Hum Genet. 2020;65(12):1075-82. https://doi.org/10.1038/s10038-020-0808-9.

37. Flores-Alanis A, Cruz-Rangel A, Rodríguez-Gómez F, González J, TorresGuerrero CA, Delgado G, et al. Molecular epidemiology surveillance of SARSCoV-2: mutations and genetic diversity one year after emerging. Pathogens. 2021;10(2):184. https://doi.org/10.3390/pathogens10020184.

38. Volz E, Hill V, McCrone JT, Price A, Jorgensen D, OToole Á, et al. Evaluating the Effects of SARS-CoV-2 Spike Mutation D614G on Transmissibility and Pathogenicity. Cell. 2021;184(1):64-75.e11.

39. Davies NG, Jarvis Cl, Edmunds WJ, Jewell NP, Diaz-Ordaz K, Keogh RH. Increased mortality in community-tested cases of SARS-CoV-2 lineage B.1.1. 7. Nature. 2021;593(7858):270-4. https://doi.org/10.1038/s41586-021-03426-1.

40. Challen R, Brooks-Pollock E, Read JM, Dyson L, Tsaneva-Atanasova K, Danon L. Risk of mortality in patients infected with SARS-CoV-2 variant of concern 202012/1: matched cohort study. BMJ. 2021;372:n579.

41. Grint DJ, Wing K, Williamson E, McDonald HI, Bhaskaran K, Evans D, et al. Case fatality risk of the SARS-CoV-2 variant of concern B.1.1.7 in England, 16 November to 5 February. Eurosurveillance. 2021;26(11):2100256.

42. European Centre for Disease Prevention and Control. Methods for the detection and identification of SARS-CoV-2 variants. 2021. Available from: https://www.ecdc.europa.eu/sites/default/files/documents/Methods-for-thedetection-and-identification-of-SARS-CoV-2-variants.pdf

43. Robins J, Hernan MA. Causal inference: what if. Boca Raton: Chapman \& Hall/CRC; 2020.

44. Glymour MM. Using causal diagrams to understand common problems in social epidemiology. In: Oakes JM, Kaufman JS, eds. Methods in Social Epidemiology. 2nd ed. San Francisco, CA: Jossey-Bass; 2017:458-492.

45. Suzuki E, Shinozaki T, Yamamoto E. Causal diagrams: pitfalls and tips. J Epidemiol. 2020;30(4):153-62. https://doi.org/10.2188/jea.JE20190192.

46. Frampton D, Rampling T, Cross A, Bailey H, Heaney J, Byott M, et al. Genomic characteristics and clinical effect of the emergent SARS-CoV-2 B.1. 1.7 lineage in London, UK: a whole-genome sequencing and hospital-based cohort study. Lancet Infect Dis. 2021;0(0) [cited 2021 May 19]. Available from: https://www.thelancet.com/journals/laninf/article/PIIS1473-3099(21)001 70-5/abstract.

47. Bager $\mathrm{P}$, Wohlfahrt J, Fonager J, Albertsen M, Yssing Michaelsen T, Holten Møller C, et al. Increased Risk of Hospitalisation Associated with Infection with SARS-CoV-2 Lineage B.1.1.7 in Denmark. Rochester: Social Science Research Network; 2021. Report No.: ID 3792894. [cited 2021 May 19]. Available from: https://papers.ssrn.com/abstract=3792 894

48. de Oliveira MHS, Lippi G, Henry BM. Sudden rise in COVID-19 case fatality among young and middle-aged adults in the south of Brazil after identification of the novel B.1.1.28.1 (P.1) SARS-CoV-2 strain: analysis of data from the state of Parana. medRxiv. 2021. https://doi.org/10.1101/2021. 03.24.21254046.

49. Qian Z, Alaa AM, van der Schaar M, Ercole A. Between-Centre differences for COVID-19 ICU mortality from early data in England. Intensive Care Med. 2020;46(9):1779-80. https://doi.org/10.1007/s00134-020-06150-y.

50. Mansournia MA, Hernán MA, Greenland S. Matched designs and causal diagrams. Int J Epidemiol. 2013;42(3):860-9. https://doi.org/10.1093/ije/ dyt083.

51. Elwert F, Winship C. Endogenous selection Bias: the problem of conditioning on a collider variable. Annu Rev Sociol. 2014;40(1):31-53. https://doi.org/10.1146/annurev-soc-071913-043455.

52. RAG subgroup testing. Aanbevelingen voor de selectie van stalen voor de sequentiebepaling van het volledige genoom in het kader van surveillance - update. 2021. Available from: https://covid-19.sciensano.be/sites/default/ files/Covid19/20210315_Advice\%20RAG_Selection\%20for\%20samples\%2 ofor\%20sequencing\%20-\%20update_NL.pdf

53. Pujadas E, Chaudhry F, McBride R, Richter F, Zhao S, Wajnberg A, et al. SARS-CoV-2 viral load predicts COVID-19 mortality. Lancet Respir Med. 2020; 8(9):e70. https://doi.org/10.1016/S2213-2600(20)30354-4.

54. Van Goethem N, Vilain A, Wyndham-Thomas C, Deblonde J, Bossuyt N, Lernout T, et al. Rapid establishment of a national surveillance of COVID-19 hospitalizations in Belgium. Arch Public Health. 2020;78(1):121. https://doi. org/10.1186/s13690-020-00505-z.

55. healthdata.be. Database COVID-19 TestResult. Available from: https://covid1 9lab.healthdata.be/datacollection/laboratorytestresult. Accessed 1 Aug 2021.

56. Meurisse M, Lajot A, Dupont $Y$ et al. One Year of Laboratory Based COVID19 Surveillance System in Belgium: Main Indicators and Performance of the Laboratories, 19 April 2021, PREPRINT (Version 1) available at Research Square. https://doi.org/10.21203/rs.3.rs-417041/v1.

57. healthdata.be. Database COVID-19 TestResults Variants. Available from: https://covid19lab.healthdata.be/laboratorytestresultvariantsAccessed 1 Aug 2021.

58. Rambaut A, Holmes EC, OToole Á, Hill V, McCrone JT, Ruis C, et al. A dynamic nomenclature proposal for SARS-CoV-2 lineages to assist genomic epidemiology. Nat Microbiol. 2020;5(11):1403-7. https://doi.org/10.1038/s41 564-020-0770-5.

59. Smith LH, Mathur MB, VanderWeele TJ. Multiple-bias Sensitivity Analysis Using Bounds. Epidemiology. 2021:32:625-34. https://doi.org/10.1097/EDE. 0000000000001380.

60. The Severe Covid-19 GWAS Group. Genomewide Association Study of Severe Covid-19 with Respiratory Failure. New England Journal of Medicine. 2020;383(16):1522-34.

61. Chakravarty S. COVID-19: the effect of host genetic variations on host-virus interactions. J Proteome Res. 2021;20(1):139-53. https://doi.org/10.1021/acs. jproteome.0c00637.

62. Hoffmann SC, Stanley EM, Cox ED, DiMercurio BS, Koziol DE, Harlan DM, et al. Ethnicity greatly influences cytokine gene polymorphism distribution. Am J Transplant. 2002;2(6):560-7. https://doi.org/10.1034/j.1600-6143.2002.2 0611.x.

63. Cao Y, Li L, Feng Z, Wan S, Huang P, Sun X, et al. Comparative genetic analysis of the novel coronavirus (2019-nCoV/SARS-CoV-2) receptor ACE2 in different populations. Cell Discov. 2020;6(1):1-4. https://doi.org/10.1038/ s41421-020-0147-1.

64. Ortiz-Fernández L, Sawalha AH. Genetic variability in the expression of the SARS-CoV-2 host cell entry factors across populations. Genes Immun. 2020; 21(4):269-72. https://doi.org/10.1038/s41435-020-0107-7.

65. López de Maturana E, Alonso L, Alarcón P, Martín-Antoniano IA, Pineda S, Piorno $L$, et al. Challenges in the Integration of Omics and Non-Omics Data. 
Genes (Basel). 2019;10(3) cited 2021 May 21]. Available from: https://www. ncbi.nlm.nih.gov/pmc/articles/PMC6471713/.

66. Munafò MR, Tilling K, Taylor AE, Evans DM, Davey SG. Collider scope: when selection bias can substantially influence observed associations. Int J Epidemiol. 2018;47(1):226-35. https://doi.org/10.1093/ije/dyx206.

67. European Centre for Disease Prevention and Control. Guidance for representative and targeted genomic SARS-CoV-2 monitoring. 2021.

68. World Health Organization. Genomic sequencing of SARS-CoV-2: a guide to implementation for maximum impact on public health. 2021 [cited 2021 Jan 17]. Available from: https://www.who.int/publications-detail-redirect/ 9789240018440

69. Brazauskas R, Logan BR. Observational studies: matching or regression? Biol Blood Marrow Transplant. 2016 Mar;22(3):557-63. https://doi.org/10.1016/j. bbmt.2015.12.005.

\section{Publisher's Note}

Springer Nature remains neutral with regard to jurisdictional claims in published maps and institutional affiliations.

Ready to submit your research? Choose BMC and benefit from:

- fast, convenient online submission

- thorough peer review by experienced researchers in your field

- rapid publication on acceptance

- support for research data, including large and complex data types

- gold Open Access which fosters wider collaboration and increased citations

- maximum visibility for your research: over $100 \mathrm{M}$ website views per year

At BMC, research is always in progress.

Learn more biomedcentral.com/submissions 